\title{
VPA and MEL induce apoptosis by inhibiting the Nrf2-ARE signaling pathway in TMZ-resistant U251 cells
}

\author{
HAO PAN ${ }^{1}$, HANDONG WANG $^{1}$, YUE JIA ${ }^{1}$, QIANG WANG ${ }^{1}$, LIWEN LI ${ }^{1}$, QI WU ${ }^{1}$ and LONGBANG CHEN ${ }^{2}$ \\ Departments of ${ }^{1}$ Neurosurgery and ${ }^{2}$ Oncology, Jinling Hospital, School of Medicine, \\ Nanjing University, Nanjing, Jiangsu 210002, P.R. China
}

Received February 22, 2016; Accepted February 23, 2017

DOI: $10.3892 / \mathrm{mmr} .2017 .6621$

\begin{abstract}
Chemoresistance is the primary obstacle to effective treatment of glioblastoma, the most lethal brain tumor. Our previous study demonstrated that Nf-E2 related factor 2 (Nrf2), a traditional cytoprotective transcription factor, was overexpressed in gliomas and promoted malignancy. The present study aimed to investigate the expression levels of Nrf2-antioxidant response element (ARE) signaling pathway genes in temozolomide (TMZ)-resistant U251 human glioblastoma cells (U251-TMZ). Additionally, the effect of valproic acid (VPA) and melatonin (MEL) on Nrf2 expression in U251-TMZ cells and their association with chemoresistance was investigated. The results of the present study indicated that the expression levels of components of the Nrf2-ARE signaling pathway were increased in U251-TMZ cells compared with U251 parent cells. Silencing of Nrf2 by transfection with small interfering RNA restored the chemosensitivity of U251-TMZ cells. The Nrf2 inhibitors VPA and MEL successfully reduced Nrf2 expression and survival in U251-TMZ cells treated with TMZ, accompanied by increased reactive oxygen species levels and apoptosis. Therefore, VPA and MEL may be potential chemotherapeutic sensitizers for the treatment of chemoresistant glioblastoma.
\end{abstract}

\section{Introduction}

Glioma accounts for 30 to $40 \%$ of all intracranial tumors. Glioblastoma, which is the most lethal primary brain tumor, is the most common malignant glioma $(1,2)$. Treatment strategies include combinations of aggressive resection, radiotherapy and temozolomide (TMZ) treatment and various

Correspondence to: Dr Handong Wang, Department of Neurosurgery, Jinling Hospital, School of Medicine, Nanjing University, 305 East Zhongshan Road, Nanjing, Jiangsu 210002, P.R. China

E-mail: njhdwang@hotmail.com

Key words: glioblastoma, Nf-E2 related factor 2-antioxidant response element, chemoresistance, valproic acid, melatonin, apoptosis novel chemotherapeutics. However, the median survival time of patients remains poor (3). Chemoresistant glioma cell subgroups are the most common cause of recurrence.

Our previous study determined that the expression of Nf-E2 related factor 2 (Nrf2), a traditional cytoprotective transcription factor, was elevated in glioma (4-8). Increased expression and continuous activation of Nrf2 in glioma may contribute to proliferation, invasion and chemoresistance of cancer cells. A previous study identified increased expression levels of Nrf2 and its target proteins in doxorubicin-resistant BEL-7402 hepatocellular carcinoma cells compared with parent cells (9). Chemoresistance was reduced in doxorubicin-resistant BEL-7402 cells following suppression of Nrf2 expression by chrysin, a potent Nrf2 inhibitor. The present study investigated the $\mathrm{Nrf2}$-antioxidant response element (ARE) signaling pathway in TMZ-resistant U251. Additionally, the effects of valproic acid (VPA) and melatonin (MEL) on Nrf2 expression levels and chemoresistance in U251-TMZ cells were investigated. The present study determined that increased expression of components of the Nrf2-ARE signaling pathway in U251-TMZ cells was reversed by VPA and MEL treatment, which act as Nrf2 inhibitors. Additionally, U251-TMZ cells restored chemosensitivity to TMZ following co-treatment with VPA or MEL and exhibited increased levels of reactive oxygen species (ROS) and apoptosis.

\section{Materials and methods}

Cell culture and TMZ resistance. Human U251 glioblastoma cells were obtained from the American Type Culture Collection (Manassas, VA, USA) and cultured in Dulbecco's modified Eagle's medium (HyClone; GE Healthcare Life Sciences, Logan, UT, USA) with $10 \%$ fetal bovine serum (Wisent Biotechnology Co., Ltd., Nanjing, China). The TMZ-resistant U251 cell line (termed U251-TMZ) was established by sustained low concentration TMZ (cat. no. T2577; Sigma; Merck KGaA, Darmstadt, Germany) stimulation of $\mathrm{U} 251$ cells, at an initial dose of $0.25 \mu \mathrm{M}$ for 2 weeks. Then, the concentration of TMZ was doubled every 2-4 weeks. Following this stepwise increase in TMZ concentration for $\sim 8$ months, the final U251-TMZ cell line was able to survive in $100 \mu \mathrm{M}$ TMZ. Subsequently, Cell Counting kit-8 (CCK-8; Dojindo Molecular Technologies, Inc., Kumamoto, Japan) was 
used to evaluate the survival of U251-TMZ cells and the parent U251 cell line following treatment with TMZ.

Transient transfection with small interfering (si)RNA. Nrf2 siRNA (si-Nrf2) (target sequence, 5'GCA GTTCAATGAAGCTCAACT3') and scramble siRNA (si-con; sequence, 5'GAGUACGAUCCGAGUUGAG3') were purchased from Guangzhou RiboBio Co., Ltd. (Guangzhou, China). U251-TMZ cells (1×10\%/well) were seeded in 6-well plates and transfected with $2 \mu 1$ Lipofectamine ${ }^{\circledR} 2000$ (Invitrogen; Thermo Fisher Scientific, Inc., Waltham, MA, USA), 100 pmol si-Nrf2 or si-con, and $2 \mathrm{ml}$ reduced serum Opti-Minimal Essential Medium (Invitrogen; Thermo Fisher Scientific, Inc.) according to the manufacturer's protocol. Cells treated with Lipofectamine 2000 only served as the blank control group. The success of the transfection was confirmed by western blot analysis $48 \mathrm{~h}$ after transfection. All further experiments using U251-TMZ/si-Nrf2 cells were performed within $48 \mathrm{~h}$ of the transfection.

CCK-8 assay. Cells (100 $\mu \mathrm{l})$ were seeded in 96-well culture plates at a density of $2 \times 10^{3}$ cells/well and cultured for $24 \mathrm{~h}$. TMZ at $0,12.5,25,50,100,200$ and $400 \mu \mathrm{M}$ was used to check the chemoresistance of U251-TMZ. Treatment with $200 \mu \mathrm{M} \mathrm{TMZ}+\mathrm{VPA}$ at $0.5,1,2$ and $5 \mathrm{mM}$ or MEL at $0.1,0.5$, 1 and $2 \mathrm{mM}$ were used to check the effect of VPA and MEL on U251-TMZ chemosensitivity. Viable cells were quantified at $0,12,24$ and $48 \mathrm{~h}$ following treatment, using a CCK-8 according to the manufacturer's protocol. Briefly, $10 \mu \mathrm{lCCK}-8$ solution was added into every well and incubated at $37^{\circ} \mathrm{C}$ for $1 \mathrm{~h}$. Subsequently the optical density of each well was measured at a wavelength of $450 \mathrm{~nm}$ using a Bio-Rad ELISA microplate reader (Bio-Rad Laboratories, Inc., Hercules, CA, USA). All measurements were performed six times. Data are presented as the mean \pm standard deviation (SD).

Reverse transcription-quantitative polymerase chain reaction $(R T-q P C R)$. Total RNA was isolated with TRIzol ${ }^{\circledR}$ (Invitrogen; Thermo Fisher Scientific, Inc.) and single-stranded cDNA was synthesized from 2 ug of total RNA with the Transcriptor First Strand cDNA Synthesis kit (Roche Applied Science, Penzberg, Germany) according to the manufacturer's protocol. The thermocycling conditions were as follows: Denaturation for $10 \mathrm{~min}$ at $65^{\circ} \mathrm{C}$, cDNA synthesis for $1 \mathrm{~h}$ at $50^{\circ} \mathrm{C}$ and inactivation for $5 \mathrm{~min}$ at $85^{\circ} \mathrm{C}$. The cDNA was stored at $-20^{\circ} \mathrm{C}$. Quantitative analysis of Nrf2 mRNA expression levels was performed using SYBR ${ }^{\circledR}$ Green PCR Core reagents (Thermo Fisher Scientific, Inc.) and an ABI 7700 Real-time system (Applied Biosystems; Thermo Fisher Scientific, Inc.), according to the manufacturer's protocol. The primer sequences were as follows: Forward, 5'-TTCCCGGTCACATCGAGAG-3' and reverse, 5'-TCCTGT TGCATACCGTCTAAATC-3' for Nrf2; and forward, 5'-CAT CTTCTTTTGCGTCGCCA-3' and reverse, 5'-TTAAAAGCA GCCCTGGTGACC-3' for GAPDH. Each amplification cycle consisted of denaturation for $15 \mathrm{sec}$ at $95^{\circ} \mathrm{C}$, annealing for $20 \mathrm{sec}$ at $60^{\circ} \mathrm{C}$ and extension for $40 \mathrm{sec}$ at $72^{\circ} \mathrm{C}$; a total of 40 cycles were performed. A melting curve was used to distinguish specific from non-specific products and primer-dimers. Each sample was analyzed in triplicate, and expression of Nrf2 was normalized to GAPDH using the $2^{-\Delta \Delta C q}$ method (10).
Alterations in Nrf2 expression were reported as fold increases $\left(2^{-\Delta \Delta \mathrm{Cq})}\right.$ relative to the control group.

Protein preparation. Cytoplasmic and nuclear proteins were obtained using the Nuclear and Cytoplasmic Protein Extraction kit (Beyotime Institute of Biotechnology, Shanghai, China). Briefly, cells were treated with cytoplasmic protein extraction reagent. Lysates were subsequently incubated for $10 \mathrm{~min}$ on ice and centrifuged at $12,000 \mathrm{xg}$ for $5 \mathrm{~min}$ at $4^{\circ} \mathrm{C}$. The supernatant was collected as the cytoplasmic protein. Pellets containing crude nuclei were resuspended in nuclear protein extraction regent and agitated for $2 \mathrm{~h}$ on ice, followed by centrifugation at $12,000 \mathrm{x}$ g for $10 \mathrm{~min}$ at $4^{\circ} \mathrm{C}$ to obtain supernatants containing nuclear protein. To obtain total protein lysate, cells were harvested and homogenized using radioimmunoprecipitation assay buffer (Beyotime Institute of Biotechnology) containing $1 \mathrm{mM}$ phenylmethylsulfonyl fluoride, and centrifuged at $12,000 \mathrm{x} \mathrm{g}$ for $15 \mathrm{~min}$ at $4^{\circ} \mathrm{C}$. Protein concentrations were estimated by Coomassie Plus Protein assay reagent (Pierce; Thermo Fisher Scientific, Inc.).

Western blot analysis. Protein extracts $(50 \mathrm{mg})$, including cytoplasmic and nuclear protein for Nrf2 detection, nuclear protein for $\mathrm{H} 3$ detection and total protein for detecting all other proteins, were heat denatured in loading buffer, separated by $10 \%$ sodium dodecyl sulfate-polyacrylamide gel electrophoresis and electroblotted onto a nitrocellulose membrane. Membranes were blocked with $5 \%$ bovine serum albumin (BSA; cat. no. B2064; Sigma; Merck KGaA) for $2 \mathrm{~h}$ at room temperature. The following primary antibodies were used: Rabbit anti-Nrf2 (68 kDa; 1:500; cat. no. sc-722; Santa Cruz Biotechnology, Inc., Dallas, TX, USA), rabbit anti-histone H3 (H3; 17 kDa; 1:2,000; cat. no. 9715; Cell Signaling Technology, Inc., Danvers, MA, USA), rabbit anti- $\beta$-actin (43 kDa; 1:10,000; cat. no. AP0060; Bioworld Technology, Inc., St. Louis Park, MN, USA), rabbit anti-HO-1 (32 kDa; 1:1,000; cat. no. ab13243; Abcam, Cambridge, UK), rabbit anti-NQO1 (31 kDa; 1:1,000; cat. no. ab34173; Abcam), rabbit anti-cleaved caspase-3 (17 kDa; 1:1,000; cat. no. 9661; Cell Signaling Technology, Inc.), rabbit anti-B-cell lymphoma 2 (Bcl-2; 26 kDa; 1:1,000; cat. no. sc-492; Santa Cruz Biotechnology, Inc.), mouse anti-Bcl-2-associated X protein (Bax; 1:500; 23 kDa; cat. no. sc-20067; Santa Cruz Biotechnology, Inc.), mouse anti-phosphorylated (p)-insulin like growth factor 1 receptor (IGF-IR; 97 kDa; 1:500; cat. no. sc-81499; Santa Cruz Biotechnology, Inc.), mouse anti-IGF-IR (97 kDa; 1:500; cat. no. sc-81167; Santa Cruz Biotechnology, Inc.), rabbit anti-protein kinase $\mathrm{B}$ (AKT; $60 \mathrm{kDa} ; 1: 1,000$; cat. no. 9272; Cell Signaling Technology, Inc.), rabbit anti-p-AKT (60 kDa; 1:2,000; cat. no. 4060; Cell Signaling Technology, Inc.), rabbit anti-mammalian target of rapamycin (mTOR; 289 kDa; 1:1,000; cat. no. 2983; Cell Signaling Technology, Inc.), rabbit anti-p-mTOR (289 kDa; 1:500; cat. no. 5536; Cell Signaling Technology, Inc.) and rabbit anti-P2X purinoceptor 7 (P2RX7; 75 kDa; 1:1,000; cat. no. ab109246; Abcam). Each primary antibody was diluted appropriately in 5\% BSA and incubated overnight at $4^{\circ} \mathrm{C}$. The blots were washed three times in washing buffer and incubated with goat anti-rabbit IgG-horseradish peroxidase (HRP; cat. no. BS13278; 1:10,000; Bioworld Technology, Inc.) or goat anti-mouse IgG-HRP 


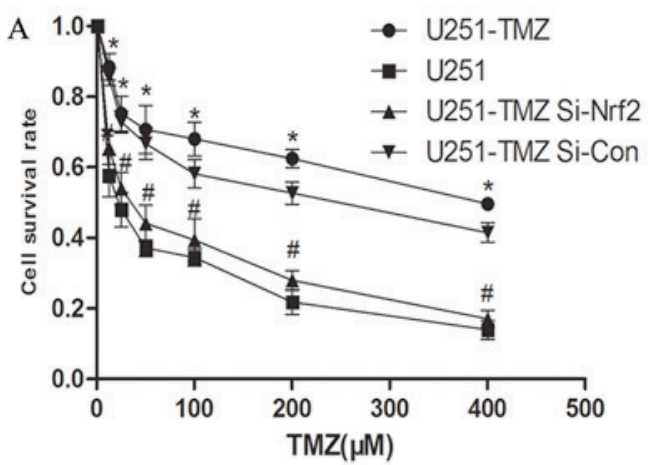

B

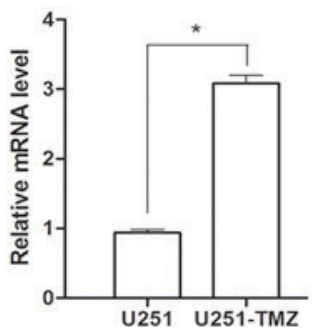

$\mathrm{C}$

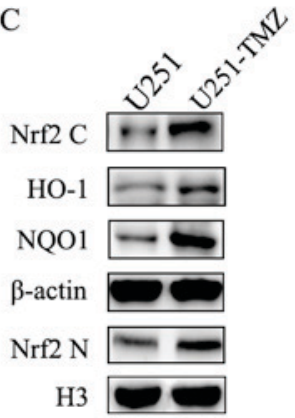

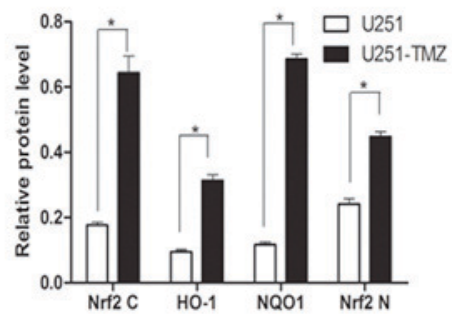

E

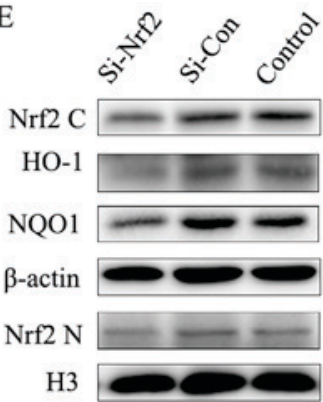

F

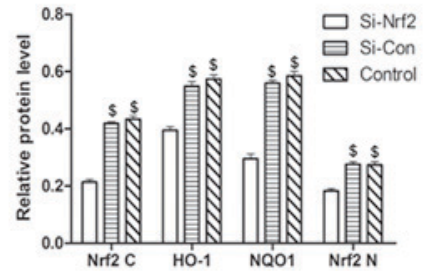

Figure 1. Nrf2-ARE signaling pathway in U251-TMZ and its association with chemoresistance. (A) Cell survival rates following treatment with different concentrations of TMZ for $24 \mathrm{~h}$. (B) Reverse transcription-quantitative polymerase chain reaction identified enhanced Nrf2 mRNA expression levels in U251-TMZ when compared with U251 cells. (C) Western blotting demonstrated elevated expression levels of Nrf2-ARE signaling pathway components in U251-TMZ compared with parent U251 cells. (D) siRNA transfection efficiency was determined by western blotting. (E) Grey scale analysis results of target proteins in U251 or U251-TMZ when compared with $\beta$-actin or H3. (F) Grey scale analysis results of target proteins in U251-TMZ following siRNA transfection when compared with $\beta$-actin or $\mathrm{H} 3 .{ }^{*} \mathrm{P}<0.05$ vs. U251; ${ }^{\circledR} \mathrm{P}<0.05$ vs. U251-TMZ; ${ }^{\$} \mathrm{P}<0.05$ vs. Si-Nrf2.Nrf2, Nf-E2 related factor 2 ; ARE, antioxidant response element; U251-TMZ, temozolomide-resistant U251; TMZ, temozolomide; siRNA, small interfering RNA; si-con, scramble control small interfering RNA; Nrf2 C, cytoplasmic Nf-E2 related factor 2; Nrf2 N, nuclear Nf-E2 related factor 2; HO-1, heme oxygenease-1; NQO1, NAD(P)H quinone dehydrogenase 1; H3, histone 3.

(cat. no. BS12478; 1:10,000; Bioworld Technology, Inc.) for $1 \mathrm{~h}$ at room temperature. Protein bands were visualized using an Enhanced Chemiluminescence Detection system (EMD Millipore, Billerica, MA, USA) and exposed by Tanon 5200 Chemiluminescence Image Analytical system (TanonScience and Technology Co., Ltd., Shanghai, China). $\beta$-actin served as a loading control for total or cytoplasmic protein; H3 served as a loading control for nuclear protein. Quantification of band density was performed using UN-SCAN-IT gel digitizing software version 6.1 (Silk Scientific, Inc., Orem, UT, USA) and data were normalized to $\beta$-actin or $\mathrm{H} 3$.

Flow cytometric analysis of apoptosis. Apoptosis was quantified using an Annexin V-Fluorescein Isothiocyanate (FITC) Apoptosis Detection kit (Beyotime Institute of Biotechnology), according to the manufacturer's protocol. The cells were washed once with incubation buffer following the aforementioned treatments, collected by centrifugation at $1,000 \mathrm{x} g$ for $5 \mathrm{~min}$ at room temperature, and resuspended in an Annexin V-FITC/propidium iodide (PI) reactive solution. Following a 15 min incubation at room temperature, the percentage of apoptotic cells was quantified using a flow cytometer (Cytomics FC500; Beckman Coulter, Inc., Brea, CA, USA) and analyzed using CellQuest Pro software version 3.3 (Mac OS X.1; BD Biosciences, San Jose, CA, USA).

ROS detection with 2',7'-dichlorofluorescein diacetate (DCFH-DA). Intracellular ROS levels were quantified using the fluorescent probe DCFH-DA (cat. no. S0033; Beyotime Institute of Biotechnology), according to the manufacturer's protocol. After $24 \mathrm{~h}$ treatment, cells were incubated with DCFH-DA in Opti-Minimal Essential Medium (Invitrogen; Thermo Fisher Scientific, Inc.) for $10 \mathrm{~min}$ at $37^{\circ} \mathrm{C}$. Subsequently, cells were washed with PBS to remove excess dye prior to quantification using the microplate reader Fluoroscan Ascent (Thermo Fisher Scientific, Inc.) with excitation and emission wavelengths of 485 and $538 \mathrm{~nm}$, respectively.

Statistical analysis. Data are expressed as the mean \pm SD and evaluated using one-way analysis of variance followed by Tukey's post hoc test. $\mathrm{P}<0.05$ was considered to indicate a statistically significant difference. All analyses were performed using SPSS version 18.0 (SPSS, Inc., Chicago, IL, USA).

\section{Results}

Establishment of the TMZ-resistant U251 cell line and protein expression levels of Nrf2-ARE signaling pathway components. The U251-TMZ cell line was established following exposure of U251 cells to continuously increasing concentrations of TMZ. CCK-8 results indicated that the survival rate was greater in in U251-TMZ cells treated with TMZ, compared with parent U251 cells (Fig. 1A).

Results from RT-qPCR (Fig. 1B) and western blotting (Fig. 1C and D) indicated that the expression levels of Nrf2 and its downstream effectors, including HO-1 and NQO1, were 

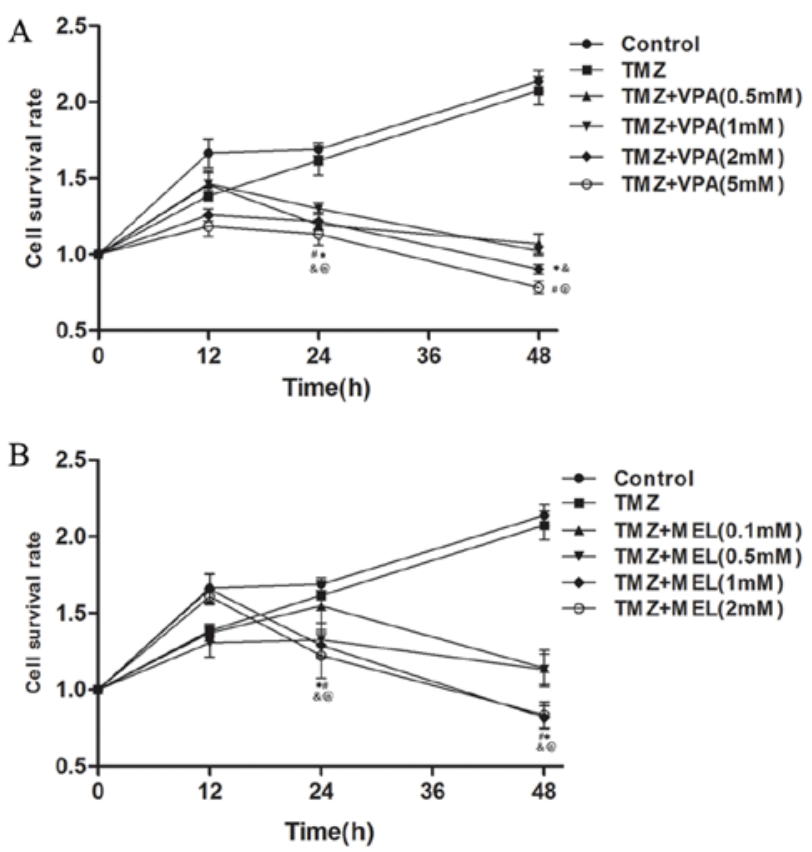

Figure 2. Survival rates of U251-TMZ cells following treatment with $200 \mu \mathrm{M}$ TMZ and VPA or MEL. (A) Survival rates of U251-TMZ cells were reduced following treatment with TMZ and VPA. "P $<0.05 \mathrm{TMZ}+2 \mathrm{mM}$ VPA and ${ }^{\text {}} \mathrm{P}<0.05 \mathrm{TMZ}+5 \mathrm{mM}$ VPA, vs. TMZ; ${ }^{\circledR} \mathrm{P}<0.05 \mathrm{TMZ}+2 \mathrm{mM}$ and ${ }^{\circledR} \mathrm{P}<0.05$ $\mathrm{TMZ}+5 \mathrm{mM}$ VPA, vs. blank control (untreated U251-TMZ cells). (B) Survival rates of U251-TMZ cells were reduced following treatment with TMZ and MEL. ${ }^{*} \mathrm{P}<0.05 \mathrm{TMZ}+1 \mathrm{mM}$ MEL and ${ }^{\#} \mathrm{P}<0.05 \mathrm{TMZ}+2 \mathrm{mM}$ MEL, vs. TMZ; ${ }^{\square} \mathrm{P}<0.05 \mathrm{TMZ}+1 \mathrm{mM}$ MEL and ${ }^{\circledR} \mathrm{P}<0.05 \mathrm{TMZ}+2 \mathrm{mM}$ MEL, vs. blank control (untreated U251-TMZ cells). U251-TMZ, temozolomide-resistant U251; TMZ, temozolomide; VPA, valproic acid; MEL, melatonin.

significantly increased in U251-TMZ cells when compared with parent $\mathrm{U} 251$ cells.

Silencing Nrf2 in U251-TMZ cells restores chemosensitivity to $T M Z$. The effect of Nrf2 silencing was determined $48 \mathrm{~h}$ after siRNA transfection using western blotting. si-Nrf2 successfully reduced Nrf2 protein expression levels in U251-TMZ cells (Fig. 1E and F). Protein expression levels of HO-1 and NQO1, downstream effectors of the Nrf2-ARE signaling pathway, were additionally decreased (Fig. 1E and F).

CCK-8 results indicated that the cell survival rate in the U251-TMZ si-Nrf2 group was significantly reduced when compared with the U251-TMZ si-con and blank control groups, almost to the level of parent U251 cells (Fig. 1A).

VPA or MEL treatment restores chemosensitivity of U251-TMZ cells. VPA and MEL have been identified as potent chemotherapeutic sensitizers in various tumor cell lines (11-14). The present study investigated whether these drugs may restore the chemosensitivity of U251-TMZ cells to TMZ. The results of the CCK-8 assay demonstrated that when used in combination with $200 \mu \mathrm{M}$ TMZ, treatment with 2 or $5 \mathrm{mM}$ VPA for 24 or $48 \mathrm{~h}$ significantly reduced the survival rate of U251-TMZ cells, when compared with TMZ treatment alone (Fig. 2A). Additionally, when U251-TMZ cells were treated with TMZ plus 1 or $2 \mathrm{mM}$ MEL for 24 or $48 \mathrm{~h}$, the survival rate was significantly reduced when compared with TMZ treatment alone (Fig. 2B).
VPA and MEL treatment increases ROS levels and apoptosis induced by TMZ in U251-TMZ cells. Apoptosis was investigated to determine the molecular mechanism underlying the inhibitory effect of $2 \mathrm{mM}$ VPA or $1 \mathrm{mM}$ MEL on $200 \mu \mathrm{M}$ TMZ-treated U251-TMZ cells for $24 \mathrm{~h}$ (Fig. 3A). The rate of apoptosis in Annexin V + PI positive cells was increased in TMZ-treated U251-TMZ cells co-treated with VPA or MEL, when compared with blank control (untreated U251-TMZ cells) or TMZ only-treated cells (Fig. 3B). Following co-treatment with TMZ and VPA/MEL, the protein expression levels of cleaved caspase- 3 and Bax were increased and those of Bcl-2 were reduced, as demonstrated by western blotting (Fig. 3D-F). Additionally, ROS levels were increased in U251-TMZ cells following co-treatment with TMZ and VPA or MEL, compared with the TMZ treatment alone or blank control groups (Fig. 3C).

VPA and MEL treatment may reduce Nrf2 expression via inhibition of the IGF-IR/AKT/mTOR signaling pathway. VPA and MEL are effective chemotherapeutic sensitizers in glioma (15-17). However, this effect and its association with Nrf2 have not been previously investigated in TMZ-resistant glioma cells. The present study determined that co-treatment of $2 \mathrm{mM}$ VPA or $1 \mathrm{mM}$ MEL with $200 \mu \mathrm{M}$ TMZ for $24 \mathrm{~h}$ significantly reduced Nrf2 protein expression levels in U251-TMZ cell lines, when compared with cells treated with TMZ only (Fig. 4A, C and D). In addition, the protein expression levels of HO-1 and NQO1, downstream effectors of the Nrf2-ARE signaling pathway, which contribute to ROS clearance, were reduced following TMZ treatment in combination with VPA or MEL, when compared with cells treated with TMZ only (Fig. 4A, C and D). Inhibition of the IGF-IR/AKT/mTOR signaling pathway by VPA or MEL treatment was determined using western blotting (Fig. 4B, E and F). Furthermore, protein expression levels of $\mathrm{P} 2 \mathrm{RX} 7$, the receptor associated with ROS generation, were increased following TMZ treatment with VPA or MEL, compared with TMZ treatment only (Fig. 4B, E and F).

\section{Discussion}

Chemoresistance reduces the chances of survival in patients with tumors, particularly in recurrent tumors. The present study investigated the possible mechanism underlying glioma chemoresistance. The findings of the current study indicated that expression levels of components of the Nrf2-ARE signaling pathway were increased in TMZ-resistant U251 cells compared with parent U251 cells. Suppressing Nrf2 expression using siRNA or the Nrf2 inhibitors VPA or MEL, restored the chemosensitivity of U251-TMZ cells to TMZ and increased the rate of apoptosis and ROS levels. Additionally, the results of the present study suggested that VPA and MEL treatment may reduce the expression levels of Nrf2-ARE signaling pathway proteins via inhibition of the IGF-IR/AKT/mTOR signaling pathway, and increase ROS levels via activation of P2RX7.

Nrf2 was initially identified as a critical transcription factor in the Kelch-like ECH-associated protein1 (Keap1)-Nrf2-ARE signaling pathway, which functions to resist oxidative stress. Under normal conditions, low constitutive quantities of Nrf2 protein are maintained by the Keap1-mediated ubiquitination 

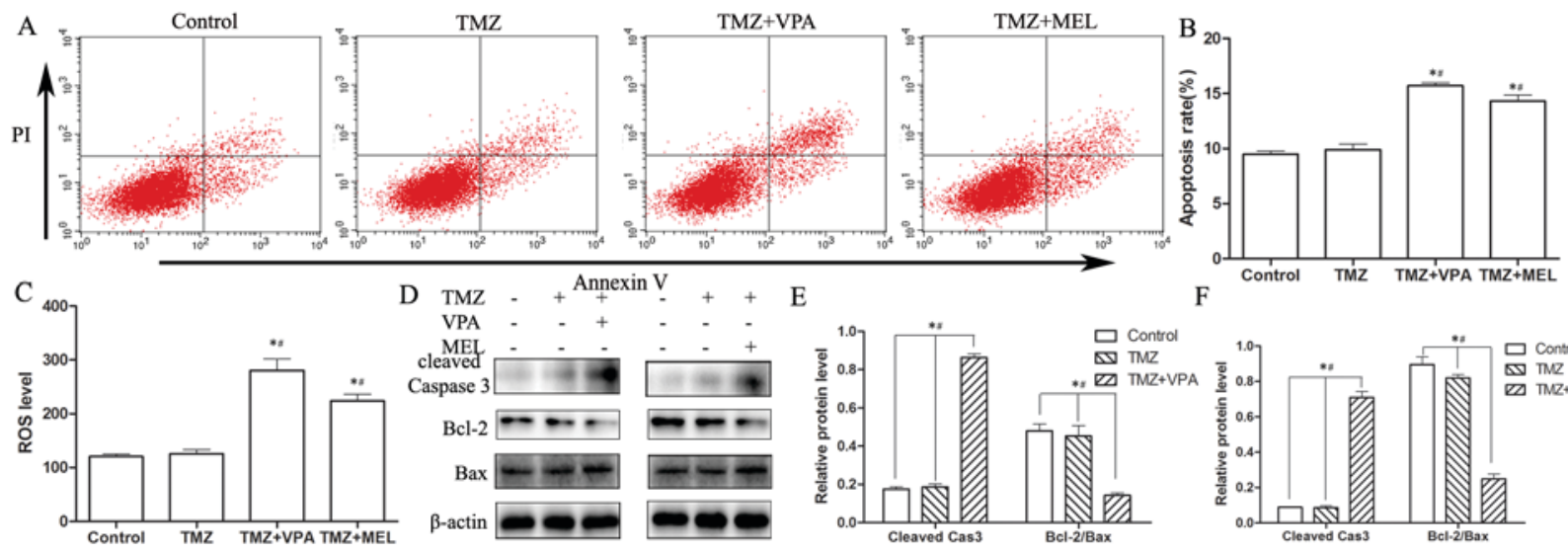

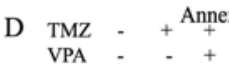
cleaved cleaved
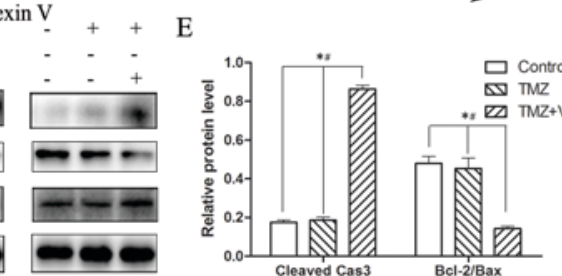
$\mathrm{F}$
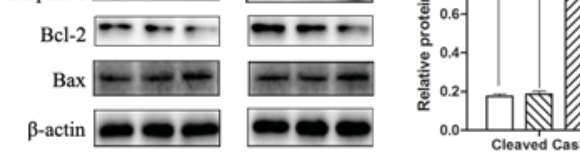

Bcl-2/Bax

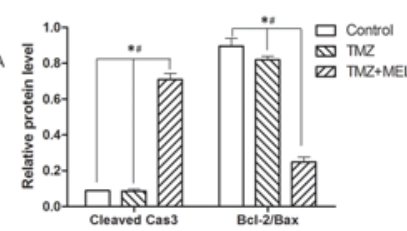

Figure 3. VPA or MEL and TMZ treatment increase apoptosis and ROS levels in U251-TMZ cells. (A) Representative plots and (B) quantification of flow cytometry indicated elevated apoptosis in U251-TMZ cells treated with TMZ plus VPA or MEL. (C) VPA or MEL co-treatment with TMZ increased ROS levels in U251-TMZ cells when compared with TMZ treatment or blank control groups. (D) Western blotting indicated increased cleaved caspase-3 and Bax, and decreased Bcl-2 protein expression levels in U251-TMZ cells treated with TMZ plus VPA or MEL. Grey scale analysis results of target protein with (E) VPA and (F) MEL treatment compared with $\beta$-actin. " $\mathrm{P}<0.05$ vs. TMZ; "P $<0.05$ vs. control (blank control with untreated U251-TMZ). TMZ, temozolomide; VPA, valproic acid; MEL, melatonin; U251-TMZ, temozolomide-resistant U251; Bcl-2, B-cell lymphoma 2; Bax, B-cell lymphoma 2-associated X protein; ROS, reactive oxygen species; PI, propidium iodide.

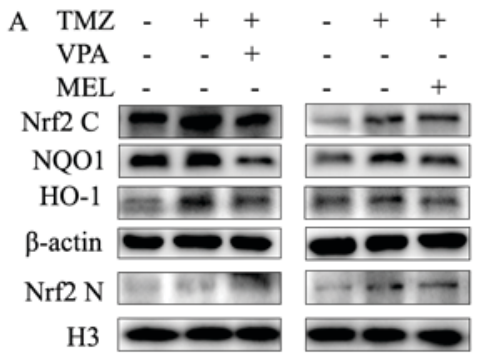

$\mathrm{D}$

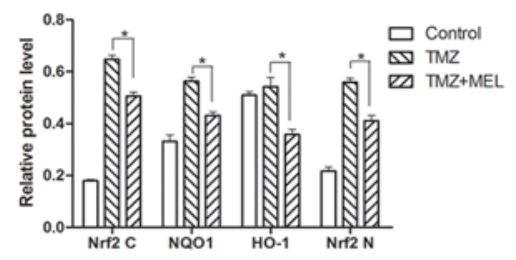

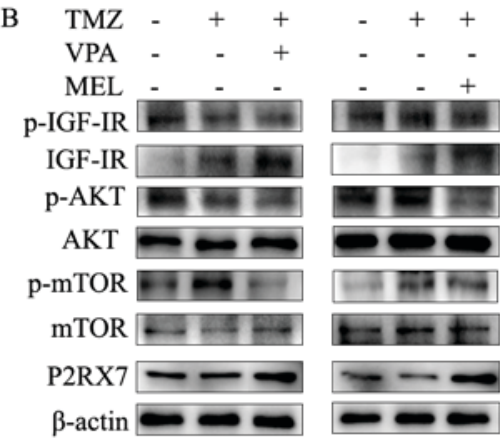

$\mathrm{E}$

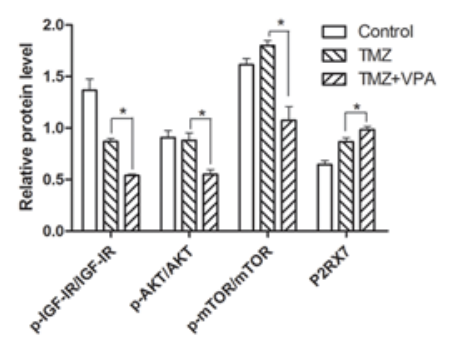

$\mathrm{C}$

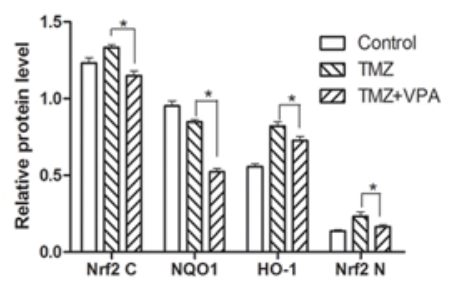

$\mathrm{F}$

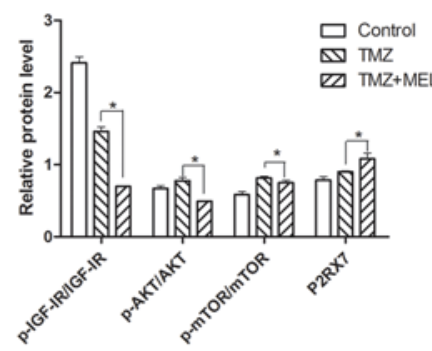

Figure 4. VPA or MEL and TMZ treatment may reduceNrf2-ARE expression via inhibition of the IGF-IR/AKT/mTOR signaling pathway and increase ROS levels via activation of P2RX7. (A) VPA or MEL co-treatment with TMZ reduced the expression levels of proteins involved in the Nrf2-ARE signaling pathway in U251-TMZ cells compared with cells treated with TMZ only. (B) VPA or MEL co-treatment with TMZ reduced phosphorylation of components of the IGF-IR/AKT/mTOR signaling pathway and increased the protein expression levels of P2RX7 in U251-TMZ cells compared with cells treated with TMZ only. Grey scale analysis results of target protein with (C) VPA and (D) MEL compared with $\beta$-actin or H3, and grey scale analysis results of phosphorylation with (E) VPA and (F) MEL compared with total protein in the IGF-IR/mTOR/AKT signaling pathway or total P2RX7 protein compared with $\beta$-actin. ${ }^{*} \mathrm{P}<0.05 \mathrm{vs}$. TMZ. Control group, blank control with untreated U251-TMZ; TMZ, temozolomide; VPA, valproic acid; MEL, melatonin; Nrf2, Nf-E2 related factor 2; ARE, antioxidant response element; U251-TMZ, temozolomide-resistant U251; Nrf2 C, cytoplasmic Nf-E2 related factor 2; Nrf2 N, nuclear Nf-E2 related factor 2; HO-1, heme oxygenease-1; NQO1, NAD(P)H quinone dehydrogenase 1; H3, histone H3; p, phosphorylated; IGF-IR, insulin like growth factor 1 receptor; AKT, protein kinase $\mathrm{B}$; mTOR, mammalian target of rapamycin; $\mathrm{P} 2 \mathrm{RX} 7, \mathrm{P} 2 \mathrm{X}$ purinoceptor 7.

and proteasomal degradation system (18). Nrf2 is activated and dissociates from Keap1 in response to oxidative stress. Following translocation from the cytoplasm to the nucleus, Nrf2 forms a heterodimer with MAF bZIP transcription factor, which binds to the ARE sequence to increase the expression of various cytoprotective genes, such as HO-1 and NQO1 (19). However, the cell protective effect of Nrf2 may additionally be present in tumor cells. Overexpression of Nrf2 accompanied by chemoresistance has been identified in non-small-cell lung cancer, breast adenocarcinoma, endometrial serous carcinoma and neuroblastoma cells (20-22). Additionally, RNA interference-mediated downregulation of Nrf2 expression in lung cancer cells induced generation of ROS and resulted in increased sensitivity to chemotherapeutic 
drugs in vitro and in vivo (23). The findings of the present study indicated increased Nrf2 expression in TMZ-resistant U251 cells compared with parent U251 cells. The protein expression levels of HO-1 and NQO1, Nrf2 targeted cytoprotective and antioxidant genes, were additionally significantly increased in U251-TMZ cells. HO-1 and NQO1 contribute to ROS clearance (24-26), which may be an important mechanism underlying TMZ-resistance. Silencing Nrf2 expression in U251-TMZ cells through siRNA transfection increased chemosensitivity to TMZ. The protein expression levels of HO-1 and NQO1 in U251-TMZ cells were reduced following si-Nrf2 transfection.

A recent study identified that VPA, a traditional antiepileptic drug, has chemotherapeutic effects (16). A meta-analysis revealed that patients with glioblastoma may experience prolonged survival following VPA administration. In addition, the benefit of VPA treatment was confirmed in sub-group analysis compared with non-anti-epileptic drug (AED) and other AED groups. These findings indicated the benefits of TMZ and VPA combined treatment in patients with glioblastoma. Previous studies have determined that VPA may inhibit glioma cell proliferation in vitro and in vivo by increasing apoptosis and inducing cell cycle arrest (27-29). The molecular mechanism underlying the effects of VPA in glioma treatment may in part rely on inhibition of histone deacetylase, downregulation of O-6-methylguanine-DNA methyltransferase, or activation of mitogen-activated protein kinases or reversion inducing cysteine rich protein with kazal motifs-matrix metalloproteinase pathways and redox regulation. However, the association between VPA and Nrf2 expression in glioma remains to be fully elucidated. The present study investigated the effect of VPA on Nrf2 expression in U251-TMZ cells. The findings of the current study demonstrated that VPA successfully downregulated the protein expression levels of Nrf2-ARE signaling pathway effectors in U251-TMZ cells. The suppression of the Nrf2-ARE signaling pathway may lead to an imbalanced redox equilibrium in the cell, which may result in elevation of ROS levels and induction of apoptosis. Therefore, inhibition of the Nrf2-ARE signaling pathway may contribute to the molecular mechanism underlying the effect of VPA treatment on glioma cells.

MEL, an indoleamine hormone produced by the pineal gland and a typical antioxidant, exerts antitumor activity in a wide range of neoplasms in vitro and in vivo (30). Martin et al (31) initially determined that MEL inhibited C6 glioma cell proliferation and induced cell cycle arrest in vitro and in vivo. Similar findings were published subsequently. Pharmacological concentrations of MEL (1 mM) may inhibit the ras oncogene at $85 \mathrm{D} /$ protein kinase $\mathrm{C} / \mathrm{AKT} /$ nuclear factor- $\kappa \mathrm{B}$ signaling pathway, which may reduce local biosynthesis of estrogen and expression of the $\mathrm{ABC}$ transporter ATP binding cassette subfamily G member 2 (32-35). These mechanisms may participate in MEL inhibition of gliomas. However, the association between the pharmacological concentration of MEL and the Nrf2-ARE signaling pathway in glioma cells remains to be fully elucidated. A previous study suggested that MEL at low concentrations (up to $100 \mathrm{nM}$ ) may activate the Nrf2-ARE signaling pathway in C6 glioma cells (36). Additionally, MEL has been revealed to activate the Nrf2-ARE signaling pathway in various neurological degeneration diseases and traumatic brain injuries $(37,38)$. However, the findings of the present study indicated that $1 \mathrm{mM}$ MEL significantly reduced Nrf2 expression and that of downstream effectors, including NQO1 and HO-1, when combined with TMZ treatment in U251-TMZ cells. The differences in these findings may be due to the different concentrations and cell types used.

Expression of Nrf2 is typically regulated by the Keap1-Nrf2-ARE signaling pathway. However, previous studies identified a role for the AKT/mTOR signaling pathway in the regulation of $\mathrm{Nrf} 2$ expression. Phosphorylation of AKT has been demonstrated to induce activation of Nrf2 and upregulate the expression of HO-1 and NQO1. Nrf2 activators, including S-allyl cysteine, berberine, oltipraz and sulforaphane, may induce the activation of the AKT signaling pathway, thus influencing the Nrf2-ARE signaling pathway (39-42). The present study demonstrated that VPA and MEL inhibited the phosphorylation of the IGF-IR/AKT/mTOR signaling pathway and inhibited the Nrf2-ARE signaling pathway. Suppression of Nrf2-ARE may cause an imbalance of the redox equilibrium, leading to increased ROS levels and apoptosis following TMZ treatment. The inhibitory effect of VPA and MEL on Nrf2 expression partly restored chemosensitivity of U251-TMZ cells. To the best of our knowledge, the present study is the first to identify that VPA and MEL maybe potential therapeutic agents for the treatment of chemoresistant glioblastoma. Further investigations are required to confirm this effect of VPA and MEL, including in vivo experiments and preclinical studies. Additionally, potential side effects due to high doses of VPA or MEL should be considered.

\section{Acknowledgements}

The present study was supported by the National Natural Science Foundation of China (grant no. 81402072), the Natural Science Foundation of Jiangsu Province (grant no. BK20140732) and the China Postdoctoral Science Foundation (grant no. 2015M572716).

\section{References}

1. Schneider T, Mawrin C, Scherlach C, Skalej M and Firsching R: Gliomas in adults. Dtsch Arztebl Int 107: 799-808, 2010.

2. Ohgaki $\mathrm{H}$ and Kleihues P: Population-based studies on incidence, survival rates, and genetic alterations in astrocytic and oligodendroglial gliomas. J Neuropathol Exp. Neurol 64: 479-489, 2005.

3. DeAngelis LM: Brain tumors. N Engl J Med 344: 114-123, 2001.

4. Pan H, Wang H, Zhu L, Mao L, Qiao L and Su X: The role of Nrf2 in migration and invasion of human glioma cell U251. World Neurosurg 80: 363-370, 2013.

5. Ji XJ, Chen SH, Zhu L, Pan H, Zhou Y, Li W, You WC, Gao CC Zhu JH, Jiang K and Wang HD: Knockdown of NF-E2-related factor 2 inhibits the proliferation and growth of U251MG human glioma cells in a mouse xenograft model. Oncol Rep 30: 157-164, 2013.

6. Pan H, Wang H, Zhu L, Wang X, Cong Z, Sun K and Fan Y: The involvement of Nrf2-ARE pathway in regulation of apoptosis in human glioblastoma cell U251. Neurol Res 35: 71-78, 2013.

7. Zhou Y, Wang HD, Zhu L, Cong ZX, Li N, Ji XJ, Pan H, Wang JW and Li WC: Knockdown of Nrf2 enhances autophagy induced by temozolomide in U251 human glioma cell line. Oncol Rep 29: 394-400, 2013.

8. Ji X, Wang H, Zhu J, Zhu L, Pan H, Li W, Zhou Y, Cong Z, Yan F and Chen S: Knockdown of Nrf2 suppresses glioblastoma angiogenesis by inhibiting hypoxia-induced activation of HIF-1 $\alpha$. Int J Cancer 135: 574-584, 2014. 
9. Gao AM, Ke ZP, Shi F, Sun GC and Chen H: Chrysin enhances sensitivity of BEL-7402/ADM cells to doxorubicin by suppressing $\mathrm{PI} 3 \mathrm{~K} / \mathrm{Akt} / \mathrm{Nrf} 2$ and ERK/Nrf2 pathway. Chem Biol Interact 206: 100-108, 2013

10. Livak KJ and Schmittgen TD: Analysis of relative gene expression data using real-time quantitative PCR and the 2(-Delta Delta C(T)) Method. Methods 25: 402-408, 2001

11. Gong Y, Ni ZH, Zhang X, Chen XH and Zou ZM: Valproic Acid Enhances the Anti-Tumor Effect of (-)-gossypol to Burkitt Lymphoma Namalwa Cells. Biomed Environ Sci 28: 773-777, 2015.

12. Fushida S, Kaji M, Oyama K, Hirono Y, Nezuka H, Takeda T, Tsukada T, Fujimoto D, Ohyama S, Fujimura T and Ohta T: Randomized Phase II trial of paclitaxel plus valproic acid vs paclitaxel alone as second-line therapy for patients with advanced gastric cancer. Onco Targets Ther 8: 939-941, 2015.

13. Sookprasert A, Johns NP, Phunmanee A, Pongthai P, Cheawchanwattana A, Johns J, Konsil J, Plaimee P, Porasuphatana S and Jitpimolmard S: Melatonin in patients with cancer receiving chemotherapy: A randomized, double-blind, placebo-controlled trial. Anticancer Res 34: 7327-7337, 2014.

14. Fan L, Sun G, Ma T, Zhong F and Wei W: Melatonin overcomes apoptosis resistance in human hepatocellular carcinoma by targeting survivin and XIAP. J Pineal Res 55: 174-183, 2013.

15. Thotala D, Karvas RM, Engelbach JA, Garbow JR, Hallahan AN, DeWees TA, Laszlo A and Hallahan DE: Valproic acid enhances the efficacy of radiation therapy by protecting normal hippocampal neurons and sensitizing malignant glioblastoma cells. Oncotarget 6: 35004-35022, 2015.

16. Yuan Y, Xiang W, Qing M, Yanhui L, Jiewen L and Yunhe M: Survival analysis for valproic acid use in adult glioblastoma multiforme: A meta-analysis of individual patient data and a systematic review. Seizure 23: 830-835, 2014.

17. Martín V, Sanchez-Sanchez AM, Herrera F, Gomez-Manzano C, Fueyo J, Alvarez-Vega MA, Antolín I and Rodriguez C: Melatonin-induced methylation of the ABCG2/BCRP promoter as a novel mechanism to overcome multidrug resistance in brain tumour stem cells. Br J Cancer 108: 2005-2012, 2013

18. Cullinan SB, Gordan JD, Jin J, Harper JW and Diehl JA The Keap1-BTB protein is an adaptor that bridges Nrf2 to a Cul3-based E3 ligase: Oxidative stress sensing by a Cul3-Keap1 ligase. Mol Cell Biol 24: 8477-8486, 2004.

19. Lau A, Villeneuve NF, Sun Z, Wong PK and Zhang DD: Dual roles of Nrf2 in cancer. Pharmacol Res 58: 262-270, 2008.

20. Jiang T, Chen N, Zhao F, Wang XJ, Kong B, Zheng W and Zhang DD: High levels of Nrf2 determine chemoresistance in type II endometrial cancer. Cancer Res 70: 5486-5496, 2010.

21. Hu L, Miao W, Loignon M, Kandouz M and Batist G: Putative chemopreventive molecules can increase Nrf2-regulated cell defense in some human cancer cell lines, resulting in resistance to common cytotoxic therapies. Cancer Chemother Pharmacol 66: 467-474, 2010

22. Wang XJ, Sun Z, Villeneuve NF, Zhang S, Zhao F, Li Y, Chen W, Yi X, Zheng W, Wondrak GT, et al: Nrf2 enhances resistance of cancer cells to chemotherapeutic drugs, the dark side of Nrf2. Carcinogenesis 29: 1235-1243, 2008.

23. Singh A, Boldin-Adamsky S, Thimmulappa RK, Rath SK, Ashush H, Coulter J, Blackford A, Goodman SN, Bunz F, Watson WH, et al: RNAi-mediated silencing of nuclear factor erythroid-2-related factor 2 gene expression in non-small cell lung cancer inhibits tumor growth and increases efficacy of chemotherapy. Cancer Res 68: 7975-7984, 2008.

24. Kuroda H, Takeno M, Murakami S, Miyazawa N, Kaneko T and Ishigatsubo Y: Inhibition of heme oxygenase-1 with an epidermal growth factor receptor inhibitor and cisplatin decreases proliferation of lung cancer A549 cells. Lung Cancer 67: 31-36, 2010.

25. Rushworth SA, Bowles KM, Raninga P and MacEwan DJ NF-kappaB-inhibited acute myeloid leukemia cells are rescued from apoptosis by heme oxygenase-1 induction. Cancer Res 70: 2973-2983, 2010

26. Kim YS, Zerin T and Song HY: Antioxidant action of ellagic acid ameliorates paraquat-induced A549 cytotoxicity. Biol Pharm Bull 36: 609-615, 2013
27. Ryu CH, Yoon WS, Park KY, Kim SM, Lim JY, Woo JS, Jeong $\mathrm{CH}$, Hou Y and Jeun SS: Valproic acid downregulates the expression of MGMT and sensitizes temozolomide-resistant glioma cells. J Biomed Biotechnol 2012: 987495, 2012.

28. Chen Y, Tsai YH and Tseng SH: Valproic acid affected the survival and invasiveness of human glioma cells through diverse mechanisms. J Neurooncol 109: 23-33, 2012.

29. Chen $\mathrm{CH}$, Chang YJ, Ku MS, Chung KT and Yang JT: Enhancement of temozolomide-induced apoptosis by valproic acid in human glioma cell lines through redox regulation. J Mol Med (Berl) 89: 303-315, 2011.

30. Srinivasan V, Spence DW, Pandi-Perumal SR, Trakht I and Cardinali DP: Therapeutic actions of melatonin in cancer: Possible mechanisms. Integr Cancer Ther 7: 189-203, 2008.

31. Martín V, Herrera F, Carrera-Gonzalez P, García-Santos G, Antolín I, Rodriguez-Blanco J and Rodriguez C: Intracellular signaling pathways involved in the cell growth inhibition of glioma cells by melatonin. Cancer Res 66: 1081-1088, 2006.

32. González A, Martinez-Campa C, Mediavilla MD, Alonso-González C, Sánchez-Barceló EJ and Cos S: Inhibitory effects of pharmacological doses of melatonin on aromatase activity and expression in rat glioma cells. Br J Cancer 97: 755-760, 2007.

33. Martín V, Herrera F, García-Santos G, Antolín I, Rodriguez-Blanco J, Medina M and Rodriguez C: Involvement of protein kinase $\mathrm{C}$ in melatonin's oncostatic effect in $\mathrm{C} 6$ glioma cells. J Pineal Res 43: 239-244, 2007.

34. Martín V,García-Santos G, Rodriguez-Blanco J,Casado-Zapico S, Sanchez-Sanchez A, Antolín I, Medina M and Rodriguez C: Melatonin sensitizes human malignant glioma cells against TRAIL-induced cell death. Cancer Lett 287: 216-223, 2010.

35. Martín V, Sanchez-Sanchez AM, Herrera F, Gomez-Manzano C, Fueyo J, Alvarez-Vega MA, Antolín I and Rodriguez C: Melatonin-induced methylation of the ABCG2/BCRP promoter as a novel mechanism to overcome multidrug resistance in brain tumour stem cells. Br J Cancer 108: 2005-2012, 2013.

36. Jumnongprakhon P, Govitrapong P, Tocharus C, Pinkaew D and Tocharus J: Melatonin protects methamphetamine-induced neuroinflammation through NF- $\mathrm{KB}$ and Nrf2 pathways in glioma cell line. Neurochem Res 40: 1448-1456, 2015.

37. Ding K, Wang H, Xu J, Li T, Zhang L, Ding Y, Zhu L, He J and Zhou M: Melatonin stimulates antioxidant enzymes and reduces oxidative stress in experimental traumatic brain injury: The Nrf2-ARE signaling pathway as a potential mechanism. Free Radic Biol Med 73: 1-11, 2014.

38. Negi G, Kumar A and Sharma SS: Melatonin modulates neuroinflammation and oxidative stress in experimental diabetic neuropathy: Effects on NF- $\mathrm{KB}$ and Nrf2 cascades. J Pineal Res 50: 124-131, 2011.

39. Lee YJ, Jeong HY, Kim YB, Lee YJ, Won SY, Shim JH, Cho MK, Nam HS and Lee SH: Reactive oxygen species and PI3K/Akt signaling play key roles in the induction of Nrf2-driven heme oxygenase-1 expression in sulforaphane-treated human mesothelioma MSTO-211H cells. Food Chem Toxicol 50: 116-123, 2012.

40. Tobón-Velasco JC, Vázquez-Victorio G, Macías-Silva M, Cuevas E, Ali SF, Maldonado PD, González-Trujano ME, Cuadrado A, Pedraza-Chaverrí J and Santamaría A: RETRACTED: S-allyl cysteine protects against 6-hydroxydopamine-induced neurotoxicity in the rat striatum: Involvement of Nrf2 transcription factor activation and modulation of signaling kinase cascades. Free Radic Biol Med 53: 1024-1040, 2012.

41. Hsu YY, Tseng YT and Lo YC: Berberine, a natural antidiabetes drug, attenuates glucose neurotoxicity and promotes Nrf2-related neurite outgrowth. Toxicol Appl Pharmacol 272: 787-796, 2013.

42. Rao J, Qian X, Li G, Pan X, Zhang C, Zhang F, Zhai Y, Wang X and Lu L: ATF3-mediated NRF2/HO-1 signaling regulates TLR4 innate immune responses in mouse liver ischemia/reperfusion injury. Am J Transplant 15: 76-87, 2015. 